Cahiers de recherches médiévales

La Figure de Jules César au Moyen Âge et à la Renaissance (II)

" her star thz scriwat huru thz hauer warit ». On trouve écrit ici comment cela s'est passé

Histoire et littérature. Quelques réflexions autour de l'Erikskrönika

\title{
Corinne Péneau
}

\section{OpenEdition \\ Journals}

Édition électronique

URL : https://journals.openedition.org/crm/2584

DOI : $10.4000 / \mathrm{crm} .2584$

ISSN : $1955-2424$

Éditeur

Honoré Champion

Édition imprimée

Date de publication : 30 juin 2007

Pagination : 277-295

ISSN : 1272-9752

Référence électronique

Corinne Péneau, « "her star thz scriwat huru thz hauer warit ». On trouve écrit ici comment cela s'est passé ", Cahiers de recherches médiévales [En ligne], 14 spécial | 2007, mis en ligne le 30 juin 2010, consulté le 15 décembre 2022. URL : http://journals.openedition.org/crm/2584 ; DOI : https://doi.org/ $10.4000 / \mathrm{crm} .2584$ 


\title{
RM
}

\author{
her star thz scriwat huru thz hauer warit \\ «on trouve écrit ici comment cela s'est passés6" \\ Histoire et littérature. Quelques réflexions autour de \\ l'Erikskrönika
}

Dans Historisk tidskrift, la revue historique suédoise, est paru en 2005 un article d'Auður Magnúsdóttir dont le titre, «L'histoire médiévale est-elle vraiment de l'histoire ? ${ }^{87}$, et le contenu évoquent des réflexions apparues dans le champ des études historiques suédoises au sujet des rares thèses menées en Suède sur l'histoire médiévale, alors qu'un grand nombre de chercheurs se consacrent aux $\mathrm{XVI}^{\mathrm{e}}$ et $\mathrm{XVII}{ }^{\mathrm{e}}$ siècles. L'origine de ce déséquilibre plonge ses racines dans le positivisme suédois $^{88}$ et les critiques très vives qui ont été formulées envers les sources littéraires médiévales, en particulier les sagas islandaises. Une frontière nette a été tracée entre d'une part les sources sérieuses (les documents de la pratique) et les sources narratives. On ne peut remettre en cause la nécessité de telles distinctions consacrées par les écoles positivistes en France comme en Scandinavie, mais on ne peut qu'être choqué par les déclarations de William Ian Miller, historien spécialiste de la faide et de l'Islande, que cite Auður Magnúsdóttir : «Les vrais historiens travaillent avec des rôles de recensement, des livres de compte, des procès-verbaux, des inventaires et des documents semblables. La littérature, on la lit pendant ses loisirs. »Cette position intenable qui revient à isoler des documents «historiques» et d'autres qui ne le seraient pas, en particulier les documents «littéraires», a été depuis longtemps remise en cause, en particulier par la première école des Annales qui a montré que ce qui faisait l'historien n'était pas la source qu'il étudie, mais le type de questionnement qu'il propose. Il n'existe donc pas de document historique au sens propre et une œuvre considérée comme littéraire peut devenir aussi un objet d'histoire. C'est du moins ce que je tenterai de montrer à travers une réflexion sur la première chronique rimée suédoise, l'Erikskrönika. Ce texte du XIV siècle, dont l'unité a parfois été remise en cause, servira d'exemple, après quelques remarques générales, sur les rapports de l'histoire et de la littérature. Il permettra de montrer

\footnotetext{
${ }^{86}$ Rolf Pipping (éd.), Erikskrönikan, enligt Cod. Holm. D2 jämte avvikande läsarter ur andra handskrifter, Stockholm, 1963, et, pour la traduction française, C. Péneau, Erikskrönika, Chronique d'Erik, première chronique rimée suédoise, Paris, Publications de la Sorbonne, 2005, vers 24. Par la suite, tous les vers cités dans cet article renverront, pour le suédois, à l'édition de Rolf Pipping et, pour le français, à la traduction.

${ }^{87}$ A. Magnúsdóttir, «Är medeltidshistoria riktig historia ? », Historisk Tidskrift, 2005, p. 275286.

${ }^{88}$ L'école de Lund dite «école de Weibull », du nom de son fondateur, très marquée par le positivisme et l'œuvre théorique des Français Langlois et Seignobos, a régné dans le domaine des études historiques jusque dans les années 1970. Voir Birgitta Odén, «Metodologisk meny », Historisk Tidskrift, 1997, p. 3-23.
}

Cahiers de Recherches Médiévales (XII $-X V^{e}$ s.), 14spé, 2007 
comment une œuvre, à travers sa forme convenue, ses clichés et ses inventions peut, malgré tout, parler à l'historien.

Il existe, dans l'histoire scandinave, un fort déséquilibre entre les documents de la pratique, relativement rares, du moins si on les compare avec les séries d'archives disponibles dans le reste de l'Occident, et les documents littéraires, parfois beaucoup plus nombreux en particulier pour l'Islande. Pourtant, les œuvres littéraires ont toujours eu un statut à part, peut-être en raison de la place conférée aux auteurs et de l'indépendance reconnue à leurs œuvres, inépuisables répertoires de sens irréductible à une époque ou à un lieu. Or, en quoi cette position peut-elle effrayer l'historien, qui travaille continuellement avec ce type de texte, en particulier la Bible, dont on ne lit pas le même texte, pourtant identique, à une époque ou une autre ? Tout texte, bien que produit dans un contexte particulier, qui lui donne son sens premier, peut faire sens loin, dans le temps et dans l'espace, de son lieu de production. De plus, comme l'exprime le paradoxe du Quichotte de Pierre Ménard, un même texte écrit dans deux contextes différents ne saurait être, malgré l'exacte similitude des mots, tout à fait le même. Rien ne pourrait, au fond, mieux justifier le travail de l'historien que cette fiction de Borges.

Mais ce qui pose problème à l'historien n'est pas tant le contenu d'une œuvre, son caractère fictionnel, ou son statut. Comme le rappelle Auður Magnúsdóttir les sagas islandaises peuvent être utilisées pour étudier, par exemple, les relations familiales, les mentalités, le statut du mariage, les réseaux, les relations entre les genres. Il existe toujours, dans les replis du merveilleux le plus échevelé, cette part de vérité anthropologique dont tout historien fait son miel. Comme l'a souligné Alain Boureau dans La papesse Jeanne, il est possible de s'intéresser à « un bel objet d'histoire, lourd de sens, léger d'existence ${ }^{89} \gg$. Le mythe, la légende, la croyance, l'imaginaire, la rumeur, la fiction, ce «régime de perception socialement réglé » comme l'a définie Marc Augép intéressent aussi l'historien. La «sociologie de l'imaginaire» dont les sociologues reconnaissent que les historiens, en particulier les médiévistes, furent parmi les premiers défricheurs ${ }^{91}$, puisent largement dans les sources littéraires. En parallèle, l'objet-livre, les genres, les modes de diffusions des textes et leur langue ont une place non négligeable dans les travaux des historiens.

Ce qui pose problème aux historiens n'est pas la littérature considérée comme événement en soi, comme genre, ou lorsqu'il s'agit d'aller glaner dans les œuvres des renseignements, mais le texte en soi, considéré dans sa complétude. Tout

\footnotetext{
${ }^{89}$ A. Boureau, La papesse Jeanne, Paris, 1993, p. 9.

${ }^{90}$ M. Augé, La guerre des rêves, Paris, 1997, p. $144:$ : Si, en effet, la fiction peut se définir comme un régime de perception socialement réglé, il s'ensuit d'une part qu'elle a une existence historique qui se traduit dans des institutions, des techniques et des pratiques et d'autre part qu'elle constitue un fait socioculturel mettant en jeu des relations d'altérité, des rapports de divers types entre les uns et les autres ».

${ }^{91}$ Voir, par exemple, G. Balandier, Le pouvoir sur scène, Paris, 2006, p. 41-42, G. Bronner, Vie et mort des croyances collectives, Paris, 2006, p. 101 et P. Legros et alii, Sociologie de l'imaginaire, Paris, 2006, p. 1-2. Les deux médiévistes souvent cités sont Georges Duby et Jacques le Goff qui ont tous deux fait de l'imaginaire un terrain de recherche en soi, utilisant les sources littéraires dans une perspective anthropologique.
} 
se passe comme si, sans cesse, le texte se trouvait traversé. Or le texte n'est pas un miroir promené sur un chemin, il n'est pas le reflet d'une réalité perdue, parce qu'il est, lui-même, une part du réel et parce que le réel ne se réduit pas aux objets et aux événements, mais est lui-même formé d'idées, de mythes, de croyances, de discours. Le texte est le lieu où ces discours et ces images se croisent et font sens. Comme le souligne Paul Veyne :

La littérature ne se réduit pas à un rapport de cause ou d'effet avec la société, et la langue ne se réduit pas davantage à un code et à de l'information : elle comporte aussi une illocution, c'est-à-dire l'établissement de divers rapports spécifiques avec l'interlocuteur ${ }^{92}$.

Comme tout discours, la littérature a partie liée avec le pouvoir, elle parle d'un certain point de vue et cherche à l'imposer. La parole elle-même et ses usages sont au cœur des rapports sociaux, au-delà même du contenu propre des discours. Rappelons ce que Michel Foucault dans L'Ordre du discours soulignait: «le discours n'est pas seulement ce qui traduit les luttes ou les systèmes de domination, mais ce pour quoi, ce par quoi on lutte, le pouvoir dont on cherche à s'emparer ${ }^{93} »$. C'est ce que Jauss traduit pour la littérature par le concept d'horizon d'attente (Erwartungshorizont), qui n'est pas simplement la réponse de l'auteur aux aspirations de ses lecteurs, mais le fait que l'expérience littéraire «oriente ou modifie sa vision du monde et par conséquent réagit sur son comportement social ${ }^{94}$ », reconnaissant ainsi que la seule manière d'abolir le contentieux entre histoire et littérature était la reconnaissance de la fonction de «création sociale » assumée par la littérature qui disqualifie, selon lui, toute esthétique de la représentation ${ }^{95}$.

On aurait pourtant tort d'éliminer ce concept de représentation, car il est une donnée fondamentale du fonctionnement du pouvoir. Représentation ne signifie pas reflet ou répétition ${ }^{96}$, mais capacité à dire, à mettre en scène. Elle est ce qui, dans le texte, contribue à la rendre efficace, à marquer les esprits, à agir ${ }^{97}$. Elle n'est pas l'image, mais ce qui la rend efficiente. Trop souvent, le texte est pour l'historien un

\footnotetext{
${ }^{92}$ P. Veyne, Les Grecs ont-ils cru à leurs mythes ?, Paris, 1983, p. 30-31.

${ }^{93}$ M. Foucault, L'ordre du discours, Paris, 1971, p. 12.

${ }^{94}$ H.R. Jauss, Pour une esthétique de la réception, Paris, 1978, p. 80.

${ }^{95} I d$., p. 88.

${ }^{96}$ K. Holzermayr, "Le 'mythe' d'Arthur: la royauté et l'idéologie», Annales E.S.C. 1984, p. 493 : «Le discours littéraire est donc véritablement un «lieu» où le monde dans son actualité historique se pense et réfléchit sur lui-même (sans se «refléter»), se représente (sans se répéter) ».

${ }^{97}$ Voir, en particulier, le rôle que Pierre Bourdieu assigne aux représentations dans le champ politique : «L'action proprement politique est possible parce que les agents, qui font partie du monde social, ont une connaissance (plus ou moins adéquate) de ce monde et que l'on peut agir sur le monde social en agissant sur leur connaissance de ce monde. Cette action vise à produire et à imposer des représentations (mentales, verbales, graphiques ou théâtrales) du monde social qui soient capables d'agir sur ce monde en agissant sur la représentation que s'en font les agents », P. Bourdieu, Ce que parler veut dire. L'économie des échanges linguistiques, Paris, 1982, p. 149.
} 
répertoire d'images qu'il se borne à glaner en surface ${ }^{98}$. Or tout l'intérêt d'une étude des représentations réside dans leur effet et dans les raisons de leur efficacité. De même, il peut être dangereux de confondre la manière dont la représentation agit et la représentation du pouvoir : une simple description des signes du pouvoir en tant que telle ne peut mener qu'à une analyse tautologique, car il est dans la nature du pouvoir de se manifester en tant que pouvoir ${ }^{99}$. Il faut se méfier tout autant des surinterprétations, de la recherche à tout prix d'un sens caché là où les signes et les rites fonctionnent bien souvent sur le registre de l'auto-représentation et non sur un mode référentiel. L'étude récente de Philippe Buc sur l'interprétation des rituels du haut Moyen Âge a mis en garde contre de tels écueils ${ }^{100}$. Les textes ne sont pas le reflet du pouvoir, ni le simple enregistrement de cérémonies où le pouvoir se met en scène, mais une manière de manifester, de revendiquer ou d'instituer le pouvoir. Dès lors, cette dimension «illocutoire» du texte apparaît comme le domaine commun à l'historien et au littéraire qui font, par exemple, d'un «système narratif ${ }^{101}$ ", de «marqueurs narratifs ${ }^{102}$ » ou encore du passage du vers à la $\operatorname{pros}^{103}$ un objet d'histoire. Dans le champs des études scandinaves, il faut citer les travaux de l'historien norvégien Sverre Bagge, en particulier l'étude qu'il a consacrée à la Sverris saga et à la Hákonar saga Hákonarsonar ${ }^{104}$.

\footnotetext{
${ }^{98}$ M. Mellberg, «Den falska vägen till representativitet. Slarvig litteraturanalys som historisk metod», Historisk Tidskrift, 2001, p. 347-366. Margaretha Mellberg montre, à partir de l'exemple de Hamlet, le danger qu'il y a, pour un historien, à tirer des citations d'un texte pour les replacer dans un contexte historique sans prêter attention au sens que leur donne leur contexte littéraire.

${ }^{99}$ P. Veyne, «Propagande expression roi, image idole oracle », L'Homme 1990, p. 7-26.

${ }^{100}$ P. Buc, The Dangers of Ritual. Between early medieval texts and social scientific theory, Princeton University Press, Princeton-Oxford, 2001, p. 9: That sources owe their being to purpose and circumstance means that historian cannot establish a linear relationship between ritual and political order.

${ }^{101}$ A. Boureau, Le système narratif de Jacques de Voragine, Paris, 1984.

102 Voir, par exemple, l'article d'Anita Guerreau-Jalabert, "L'essart comme figure de subversion de l'ordre spatial dans les romans arthuriens », Campagnes médiévales. L'Homme et son espace. Étude offertes à Robert Fossier, dir. É. Mornet, Paris, Publication de la Sorbonne, 1995, p. 59-72.

${ }^{103}$ Gabrielle Spiegel a montré comment, en France, au début du XIII ${ }^{\mathrm{e}}$ siècle, la naissance d'une historiographie en prose a constitué un mode de légitimation pour l'aristocratie laïque. C'est elle qui a formulé la notion de «logique sociale des textes»: «My focus (...) is on what might be called the social logic of the texts, in the dual sens of their relation to their site of articulation - the social space they occupy, both as products of a particular social word and as agents at work in that world - and to their discursive character as articulated 'logos', that is as literary artifacts composed of language and thus requiring literary (formal) analysis.» G. Spiegel, Romancing the Past : the Rise of Vernacular Prose Historiography in thirteenth Century France, Berkeley-Los Angeles, 1993, p. 9.

104 «My perspective is that of a historian, not in the sense that I am primarily interested in the truth of the information they contain, but in the sense that I want to use them as evidence for attitudes towards kingship and ideas of society in the leading circles of Norway in the
} 
La Suède n'a pas connu comme l'Islande et la Norvège une importante rédaction de textes littéraires en prose, mais à partir du XIV ${ }^{\mathrm{e}}$ siècle et principalement $\mathrm{au} \mathrm{XV}^{\mathrm{e}}$ siècle, furent rédigées plusieurs chroniques rimées qui permettent de suivre l'histoire du pays des années 1220 à la fin du XV siècle. Un grand nombre d'études ont été consacrées à ces chroniques, en particulier à la première et à la plus célèbre d'entre elles, l'Erikskrönika, qui a reçu son nom au XIX ${ }^{\mathrm{e}}$ siècle de son principal héros, le duc Erik Magnusson (1282-1318), fils du roi Magnus Ladulås, frère du roi Birger et père du roi Magnus Eriksson. L'Erikskrönika a été étudiée par des philologues et des spécialistes de littérature médiévale qui s'accordent à y reconnaître la plus importante œuvre littéraire du Moyen Âge suédois, œuvre dont la langue permet de définir l'ancien suédois dit classique. Les historiens ont aussi beaucoup écrit sur l'Erikskrönika, principalement pour tenter de dater l'œuvre, sans que soient jamais formulés des arguments permettant de resserrer avec certitude l'intervalle entre 1320 et 1335 , pour dresser un portrait hypothétique de son commanditaire et de son auteur ${ }^{105}$, pour identifier ou reconstruire de possibles sources et établir la fiabilité des informations qu'elle contient.

La chronique a souvent été transformée en habit d'Arlequin : la variété des épisodes, qui mêle récits de croisades vers l'est et conflits entre les rois et certaines factions de l'aristocratie, qu'il s'agisse des vrais Folkungar ou des partisans des ducs Erik et Valdemar, ou le fait que certains épisodes soient manifestement mal situés dans la chronologie, par exemple le fait que l'exécution du fils du roi Birger (1320) soit placée avant l'élection du roi Magnus Eriksson (1319) qui clôt la chronique, ont pu conduire à formuler des hypothèses sur des ajouts successifs, une compilation de sources plutôt qu'un véritable texte. De manière significative, le commentaire de l'Erikskrönika publié en 1927 par Rolf Pipping, qui a réalisé l'édition critique de la chronique, se présente lui-même comme un texte éclaté, une suite de remarques stylistiques, d'identifications de personnages, de mises en parallèle avec les sources contemporaines réalisées vers à vers, ou pour les mises en perspectives historiques, épisode par épisode. Il a fallu attendre l'essai particulièrement stimulant d'Ingvar Andersson pour qu'un historien remarque la cohérence de l'ensemble, en soulignant, par exemple, la récurrence de certains vers et la signification de leurs variations. Une interprétation globale de la chronique a pu dès lors être tentée, par exemple par Sven Jansson, qui interprète l'œuvre comme un miroir, un manuel de courtoisie, destiné à l'aristocratie suédoise ou, plus récemment par Gisella Vilhelmsdotter, qui fait de la chronique un instrument de propagande en faveur de l'élection de Magnus Eriksson, toutes les élections décrites dans la chronique apparaissant comme de simples préfigurations de l'élection finale ${ }^{106}$. Roger Andersson dans son compterendu pour Historisk Tidskrift ${ }^{107}$, se demande s'il s'agit là d'une «bonne histoire », soulignant en particulier la datation trop précoce proposée par Gisela Vilhelmsdotter

thirteenth century ». S. Bagge, From Gang Leader to the Lord's Anointed: Kingship in Sverris saga and Hákonar saga Hákonarsonar, Odense, 1996, p. 9.

105 I. Andersson, Erikskrönikans författare, Svenska akademiens minnesteckningar, Stockholm, 1958.

${ }^{106}$ G. Vilhemsdotter, Riddare, bonde och biskop, studier kring tre fornsvenska dikter jämte två nyeditioner, Stockholm Studies in History of Literature 42, Stockholm, 1999, p. 63-86.

${ }^{107}$ R. Andersson, «En god historia ?», Historisk Tidskrift, 2001, p. 221-228. 
pour faire correspondre l'œuvre et l'élection qu'elle est censée justifiée, et remettant surtout en cause la possibilité d'interpréter l'Erikskrönika comme un tout. L'absence de manuscrit du $\mathrm{XIV}^{\mathrm{e}}$ siècle et le caractère indéniablement incomplet du texte parvenu jusqu'à nous, manifesté par le nombre impair de vers conservés (4543) et par une possible lacune, ne peuvent cependant être des arguments suffisants pour disqualifier une entreprise d'interprétation globale. L'argument de l'hétérogénéité des épisodes rapportés n'est pas plus recevable, car elle constitue souvent une caractéristique des œuvres médiévales, mais surtout car, malgré leurs diversités, les épisodes de l'Erikskrönika relèvent d'une construction cyclique: à chaque génération, ses héros, les chevaliers suédois, évoluent dans des situations semblables, principalement les banquets, les guerres civiles, les croisades vers l'est, le retour à la cour et au banquet commun étant souvent l'occasion de marquer un changement de roi. En soi, le récit fonctionne et peut se lire en continu sans que, en l'absence de preuve formelle d'une rédaction qui se serait faite en plusieurs étapes très espacées dans le temps ${ }^{108}$ et quels que soient les éléments remployés par l'auteur ${ }^{109}$, sa cohérence puisse être remise en cause. Même la lacune temporelle (la chronique ne décrit pas les années 1314 à 1317) peut passer inaperçue dans une œuvre qui date les épisodes selon le jour ou la saison, jamais par année, et où il ne manque aucun élément au lecteur pour suivre le cours des événements. Les arguments logiques donnés par les historiens, par exemple au sujet de l'épisode important de l'exécution de Magnus Birgersson, dont la mort est annoncée au vers 4394 avant d'être décrite aux vers suivants et qui, surtout, est mal placée chronologiquement, ne peuvent véritablement être retenus. Le fait que l'épisode ait disparu dans la version raccourcie ${ }^{110}$ ne constitue pas non plus la preuve d'un ajout plus tardif. Aucun argument ne permet de nier qu'il a pu exister, après 1320, un texte

\footnotetext{
${ }^{108}$ La division en plusieurs étapes de rédaction s'appuie sur des arguments souvent subjectifs, des auteurs pouvant proposer des découpages et des dates de rédaction différentes. Voir, par exemple, C. Cederschiöld, Om Erikskönikan. Ett historiskt epos från Folkungatid, Göteborg, 1899 , p. 242 (texte en trois parties, avec une description des années 1317-1319 écrite en premier, sous forme de pamphlet contre le roi Birger, puis les événements précédents jusqu'en 1313 auraient été ajoutés par la suite, de même que l'épisode, mal placé chronologiquement, de l'exécution de Magnus Birgersson en 1320. Voir la discussion par R. Pipping, op. cit., p. 782-783), H. Toldberg, «Erikskrönikans omarbejdelse og fortsættelse », Arkiv för Nordisk Filologi, 1962, p. 125-197 (texte rédigé en deux parties avant et après 1302, avec des ajouts plus tardifs), Bj. Beckman, Matts Kättilmundsson och hans tid, II. 1319-1326, Stockholm, 1954, p. 366-373 (il a pu exister une chronique écrite pour Abjörn Sixtensson (mort en 1310), drots du duc Erik qui aurait été reprise pour le drots Mats Kättilmundsson, commanditaire de la chronique), et Kj. Kumlien, Historieskrivning och kungadöme i svensk medeltid, Stockholm, 1979, p. 82-119 (contrairement à Rolf Pipping, Kjell Kumlien pense que le texte le plus authentique de la chronique, rédigé selon lui en 1331 pour la majorité du roi Magnus Eriksson, est la version, plus courte, incorporée à la Grande chronique rimée $\mathrm{au} \mathrm{Xv}^{\mathrm{e}}$ siècle, version pourtant conservée dans une langue modernisée).

${ }^{109}$ L'argument d'anciennes ballades, aujourd'hui perdues, qui auraient pu être incorporées dans le texte a souvent été avancé, en particulier pour l'épisode de la femme du jarl Johan, qui tue les pirates estoniens pour venger son mari (vers 491-507).

${ }^{110} \mathrm{Kj}$. Kumlien, op. cit., p. 86-91.
} 
dont quelques manuscrits de la seconde moitié $d u X V^{\mathrm{e}}$ siècle ont conservé une forme assez satisfaisante et qui, malgré ses apparentes incohérences, mérite d'être appréhendé dans sa totalité et non comme un sac où l'historien serait autorisé à étudier des éléments divers, rassemblés là par les hasards du temps.

Lire l'Erikskrönika comme un ouvrage purement historiographique ne peut que conduire l'historien à en souligner les incongruités, les erreurs et les insuffisances. Bien que la chronique décrive un siècle d'histoire suédoise, elle est aussi, en elle-même, un curieux objet d'histoire. Son auteur n'était pas un historien et plutôt que de souligner ses erreurs, il faut comprendre les choix qu'il a opérés, les enjeux qui l'ont motivé. Lorsque de telles approches ont été tentées, l'Erikskrönika s'est trouvée réduite à une œuvre de propagande. Mais quelle œuvre ne possède pas une dimension idéologique? Par ailleurs, était-il nécessaire d'écrire une chronique aussi longue pour seulement justifier l'élection de Magnus Eriksson? Plus intéressant sans doute est de s'interroger sur les raisons qui ont conduit à décrire l'histoire suédoise à la manière d'un roman courtois. C'est en étant attentif aux choix formels, à leur sens dans la Suède de la première moitié du XIV ${ }^{\mathrm{e}}$ siècle qu'il sera possible de comprendre pourquoi les Suédois ont commencé à écrire leur histoire ${ }^{111}$.

Si l'on croit les derniers vers de Herr Ivan, l'adaptation suédoise de Ivain de Chrétien de Troyes, la reine Eufemia de Norvège (1270-1312) aurait fait traduire ce roman du français en $1303^{112}$. En tout, ce sont trois «romans», aujourd'hui connus sous le nom de «chansons d'Eufemia» (Eufemiavisorna), qui auraient été traduits sous son égide, si l'on ajoute Hertig Fredrik av Normandie, dont l'original est perdu, et Flores och Blanzeflor (Floire et Blancheflor) traduits respectivement en 1308 et en 1311. La reine de Norvège aurait ainsi voulu saluer les fiançailles du duc Erik avec sa fille Ingeborg. Eufemia et bien sûr Erik sont des figures marquantes de l'Erikskrönika qui emprunte aux romans courtois non seulement leur forme rimée, mais aussi leurs thèmes comme l'aventure (äventyr), les tournois (torney), la courtoisie (tukt). Le narrateur cite, au passage, Gauvain et Perceval en modèles parfaitement imitables ${ }^{113}$, et va jusqu'à affirmer :

Je ne connais pas de meilleurs chevaliers

dans toute la chrétienté

que ces nobles d'Uppland,

d'Östergötland et du Småland ${ }^{114}$.

\footnotetext{
${ }^{111}$ Avant l'Erikskrönika, il n'existait que des listes, de rois, de lagmän ou d'évêques. Des annales latines ont été copiées dans les monastères à partir du milieu du XIII ${ }^{\mathrm{e}}$ siècle, mais la plupart des annales rédigées en Suède dépendent en partie de l'Erikskrönika. Voir G. Paulsson, Annales suecici medii avi. Svensk medeltidsannalistik, Lund, 1974.

${ }^{112}$ E. Noreen (éd.), Herr Ivan Lejon-Riddaren, SSFS, 50, Stockholm, 1930-1931, p. 407-408. ${ }^{113}$ Vers 1395

${ }^{114}$ Vers 2718-2721 : bätra riddarskap jak ey weyt / jnnan all cristenheth / Thz er hoffmen aff vplandum / aff östergötland ok smalandum.
} 
Pour que la liste des œuvres connues soit complète pour le début du XIV ${ }^{\mathrm{e}}$ siècle, il faut mentionner un autre texte rimé rédigé en 1335, le petit prologue de la Loi de Trögd, une région d'Uppland sur laquelle s'applique un droit fiscal particulier:

Que Dieu récompense les seigneurs qui ont gouverné la Suède et qui ont défendu ce droit de bon gré.

Hélas! Quel grave préjudice

a touché la Suède à travers les deux frères,

sire Erik et Valdemar,

qui était son plus jeune frère,

deux illustres princes et nobles seigneurs !

Il se trouve, de plus, qu'ils sont morts innocents.

(...)

Maintenant, Dieu dans le royaume céleste

a accordé sa grâce à la Suède

en nous envoyant un jeune homme

qui s'appelle le roi Magnus ${ }^{115}$.

Ces vers font allusion aux thèmes les plus marquants de l'Erikskrönika, la mort des ducs Erik et Valdemar, qui après avoir été invités par leur frère, le roi Birger, à un banquet, ont été faits prisonniers et sont morts de faim (épisode célèbre en Suède sous le nom de «Banquet de Nyköping») et l'élection de Magnus, le fils d'Erik porté au pouvoir par les partisans des ducs qui se sont révoltés contre le roi Birger. Il est difficile de savoir s'il s'agit là d'une allusion directe à l'Erikskrönika ou de l'expression d'une mémoire commune ${ }^{116}$, mais il reste indéniable qu'un événement s'est produit qui a conduit à l'utilisation du modèle des romans courtois au profit d'Erik et de son fils. Cet événement n'est pas seulement la trahison de Birger et sa destitution, mais l'émergence d'une élite de propriétaires qui se définit comme une

\footnotetext{
${ }^{115}$ B. Lundberg, Tröghbolag : skogshushållning, territoriel indelning och skatt $i$ Trögd under medeltiden, Uppsala, 1952, p. 30-31 : Gudh löne them herrom i swärike bodho / thenne räät hafua störkt medh allo / Awi huat een högheliken scadha / swärike fik om thöm brödher badha / Härtik erik ok Waldemar / som hans yngxtebrodher war / Twe dyre första oc edhelä herra / the dödho oskylloghe thy är wärra (...) Nu hafuir gudh jnnan hymmerik / nadhelikit giort medh swärik / Oc hafuir oos sänt än vngan man / magnus konwnger hetir han.

${ }^{116}$ B. Lundberg, op. cit., p. 60-67.
} 
chevalerie $^{117}$ et cherche à s'approprier les modèles chevaleresques venus du continent $^{118}$.

L'Erikskrönika présente dès le prologue un véritable programme : le cadre est celui d'un banquet où assisteraient de nombreux chevaliers auxquels on demande d'écouter un récit sur la manière dont les chevaliers suédois ont vécu. Le récit évoque à plusieurs reprises des fêtes chevaleresques qui renvoient à la situation d'énonciation du récit: tout se passe donc comme si le chroniqueur tendait aux chevaliers un miroir à travers les années, mais un miroir sans fonction didactique, puisque l'auteur décrit les comportements comme courtois, sans jamais véritablement expliciter ce que signifie ce terme. La chronique fonctionne plutôt sur le mode de la tautologie, c'est-à-dire de la connivence avec un auditoire capable de comprendre les modèles qu'on lui propose. La chronique repose ainsi sur un nombre limité de clichés qui définissent une norme sociale : la supériorité des chevaliers se manifeste par une reconnaissance commune de ce dont chacun a droit. Ces convenances sont exprimées dans la chronique par l'expression récurrente som [honom] till retta baar qui peut se traduire par «comme il convient» ou «comme il en a le droit», voire «selon son rang». Elle peut signifier que l'on agit conformément à son rang ou au rang de ceux qui sont présents. Il s'agit d'une norme qui n'a pas toujours besoin de s'exprimer et qui fait référence à un ensemble de droits ou de convenances qui correspondent au statut social des chevaliers.

Selon les cas, elle peut désigner le droit à une suite à cheval importante, droit qui n'a été codifié que plus tard, au milieu du $\mathrm{XIV}^{\mathrm{e}}$ siècle, dans la Loi nationale:

Le duc chevaucha vers la Norvège.

Le drots Abjörn était avec lui

ainsi que des chevaliers comme son rang le lui permettait ${ }^{119}$.

Il faut souligner qu'il s'agit de la première description de ce type en Suède. À travers ces convenances exprimées par des topoi et revendiquées comme des droits, la chronique exprime et crée peut-être en partie les normes par lesquelles cette élite se distingue.

Parmi ces normes, le banquet et l'accès à une nourriture abondante et à des boissons de luxe, tel le vin, fournissent à la chronique une thématique d'autant plus signifiante que ses deux personnages principaux, les ducs Erik et Valdemar, sont

${ }^{117}$ Le statut d'Alsnö de 1280 a accordé une exemption d'impôt à toute personne pouvant servir le roi à cheval. Ce statut, dont ne peut bénéficier qu'une élite de propriétaires terriens, ne définit toutefois pas une noblesse au sens strict dans la mesure où les privilèges ne sont pas héréditaires et où, même dans les familles aristocratiques, il arrivait que les cadets n'en bénéficient pas. Voir S.-U. Palme, «Les impôts, le statut d'Alsnö et la formation des ordres en Suède. (1250-1350) », Problèmes de stratification sociale. Actes du Colloque International (1966), dir. R. Mousnier, Paris, 1968, p. 55-71.

${ }^{118}$ Voir C. Péneau, «Élites suédoises et modèles occidentaux dans la Grande Chronique rimée », Les élites nordiques et l'Europe occidentale XII ${ }^{e} X V^{e}$ siècles, dir. É. Mornet, Paris, 2007 (à paraître), p. 227-248.

${ }^{119}$ Vers 1847-1849 : ok hertogen til norigis reedh / Drotzet abiörn med honom war / ok herra the honom til retta baar. 
morts de faim. L'auteur décrit à plusieurs reprises des banquets, véritable lieux communs au sens où ils réunissent dans un cadre d'ordre et de paix l'ensemble des chevaliers ${ }^{120}$, mais aussi au sens où leurs descriptions reposent sur des clichés qui ne font que souligner leur caractère normatif. Cette norme récurrente atteint son expression la plus achevée dans la fête organisée par le duc Erik pour son mariage. L'exceptionnelle générosité du duc est soulignée pour justifier le surnom de «Magnanime » qui lui est donné :

Les plats étaient bons et la joie immense.

On obtenait tout ce dont on avait besoin.

Le vin et l'hydromel coulaient à flots

pour tous ceux qui étaient présents ${ }^{121}$.

L'auteur n'hésite pas à forcer le trait de sa démonstration lorsqu'il décrit la manière dont les prisonniers sont traités. Ainsi lorsque le roi Valdemar est fait prisonnier, il garde des conditions de vie décentes :

Ils l'enfermèrent dans la forteresse de Nyköping,

sans fer et sans chaîne,

et ils lui fournirent ce dont il avait besoin.

(...)

Pour les vêtements et la nourriture, ils le servaient bien.

On ne le fit pas mourir de faim ${ }^{122}$.

Ce traitement est aussi réservé à un autre prisonnier important, Erik Valdemarsson, fils du roi déchu :

Ils le conduisirent à Stockholm et l'y installèrent

en haut de la tour du château

et ils le laissèrent aller libre et détaché

et firent garnir sa table.

Ses mets étaient préparés dans la cuisine du roi :

la même nourriture que prenait le roi

était toujours servie à sa table

avec de la joie et de beaux discours.

La même boisson que l'on portait au roi

\footnotetext{
${ }^{120}$ Comme l'a montré Anita Guerreau-Jalabert, le partage de nourriture est «l'une des expressions symboliques de la communication entre les hommes, des relations sociales » et la littérature arthurienne, à travers la Table Ronde, en montre l'expression la plus achevée. A. Guerreau-Jalabert, «Aliments symboliques et symbolique de la table dans les romans arthuriens $\left(\mathrm{XII}^{\mathrm{e}}-\mathrm{XIII}{ }^{\mathrm{e}}\right) »$, Annales E.S.C., mai-juin 1992, p. 575.

${ }^{121}$ Vers 3608-3611 : ther war goder rätter ok starker friidh / man fik ther hwat man torffte wiidh / wiin ok miodh war ther ospart / widh hwario menniskio ther war.

${ }^{122}$ Vers 1283-1285 et 1298-1299: a nyköpungs hws lotho the gangen / Osmiddan ok vtan häfft / ok fingo honom huat han gaat kräfft (...)mz klede ok kost the pläghadin wäl / ekke swälte man honom i häll.
} 


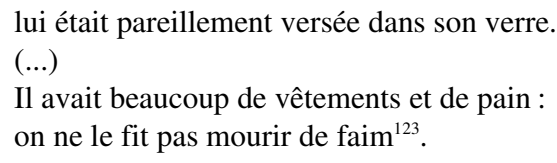

La position symbolique «en haut de la tour» et l'insistance du texte sur l'égalité entre la table royale et la table du prisonnier montrent qu'Erik est encore respecté pour ce qu'il est, un membre de la famille royale. En traitant bien les deux prisonniers, les chevaliers qui assurent la régence après la mort du roi Magnus Lådulås apparaissent comme les garants d'un système de valeurs qui reconnaît à chacun sa position et qui lie, par exemple, l'état de chevalier à l'accès à une nourriture abondante et à de riches vêtements.

Au contraire, le roi Birger se comporte de manière scandaleuse lorsqu'il fait emprisonner ses frères. Ce n'est pas la trahison qui choque le narrateur, bien qu'il évoque la figure de Judas ${ }^{124}$, car ces épisodes de capture sont nombreux dans la chronique, et les ducs eux-mêmes se sont emparés du roi Birger comme celui-ci le rappelle au moment de l'arrestation d'Erik et Valdemar ${ }^{125}$. Le roi avait été libéré contre le serment de respecter le partage du pouvoir entre lui et ses frères en Suède. Ce qui est compris comme une véritable trahison est que le roi puisse revenir sur son serment et surtout qu'il brise la norme sociale. Ainsi, l'auteur souligne que les ducs furent emprisonnés dans la «salle la plus basse ${ }^{126}$ » de la forteresse de Nyköping, puis décrit les conditions de détentions des ducs, les tortures subies, les entraves et les chaînes qui les empêchent de bouger et rappelle enfin :

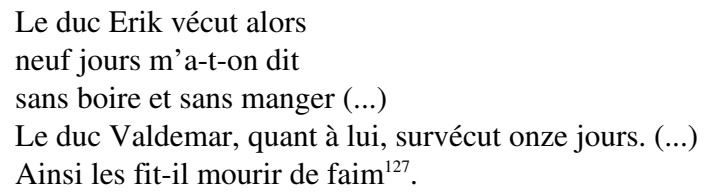

Comme le fit remarquer Ingvar Andersson, ce dernier vers crée une rupture dans le retour régulier du topos «on ne le fit pas mourir de faim», mais le procédé ne se

\footnotetext{
${ }^{123}$ Vers 1308-1317 et 1320-1321: The fördon til stokholm oc satton ther / vpa thz tornith $i$ hwsit stär / Ok lotho han lidughan ok lösan gaa / ok lotho hans disk fulwäl staa / $i$ konungsins stekerhws redhis hans maat / ä tholik kost som konungen aat / Tha gaff man honom a hans bord / ther til gläde ok fagher ord / Tholik dryk man konungenom baar / slikt sama loot man $i$ hans kaar / yffrit hade han kläder ok brödh / ekke swälte man honom til dödh.

${ }^{124}$ Vers 3633,3767 et 3769.

${ }^{125}$ Voir le très célèbre vers 3864, « Mynnes jder nokot aff hatwna leek / Vous souvenez-vous du coup de Håtuna ?» : à Håtuna, domaine situé au nord du lac Mälaren, le roi Birger et la reine avaient été fait prisonniers en 1306.

${ }^{126}$ Vers 3936.

${ }^{127}$ Vers 4060-4062, 4064 et 4067-4068 : Tha haffde hertogh Erik / nyo dagha swa sagde man mik / vtan drik ok vtan math (...) Ok ällowo dagha hertogh waldemar (...)Tessalund swälte han them $i$ häll.
} 
résume pas à un simple effet littéraire ou une volonté d'effacer les anciens conflits ${ }^{128}$. La cohérence littéraire a pour corollaire la construction d'une norme. L'auteur ne s'est pas contenté de traduire le choc ressenti par les partisans des ducs à l'annonce de leur mort: il a donné du sens à cette indignation en construisant un récit où l'épisode se trouve interprété en fonction de l'image idéale du chevalier. C'est en se référant aux clichés courtois qu'il tente de faire prendre conscience à son auditoire des implications politiques, voire juridiques, de cet acte, justifiant ainsi l'exil du roi Birger et l'exécution de son fils.

Les vers qui décrivent l'exécution de Magnus Birgersson sont placés juste avant l'élection de Magnus Eriksson. Les deux scènes forment un véritable diptyque: à travers Magnus, c'est l'héritier de Birger et donc toute idée de succession héréditaire qui se trouve écartée, tandis que l'élection du jeune Magnus Eriksson permet à ceux que la chronique nomme les «Upplandais» de montrer leur attachement à la loi élective. Le terme d'Upplandais désigne dans la chronique les chevaliers suédois favorables à l'élection du roi, dans la mesure où les Upplandais ont été les premiers à rédiger, en 1296, une loi provinciale décrivant l'élection royale ${ }^{129}$. Mais évoquer la captivité et la mort de Magnus Birgersson permet également de rétablir symboliquement les conventions bafouées par Birger. L'homme qui vient prévenir le jeune homme qu'il doit se préparer à mourir lui rappelle :

«Comme j'ai entendu les sires le dire,

tu dois expier le crime de ton père ${ }^{130} »$.

De façon paradoxale, l'exécution de Magnus est justifiée par la loi (il a pris les armes contre les représentants du royaume, ce que la Loi d'Uppland punit de mort), mais Magnus apparaît lui-même comme innocent, poussé à prendre les armes par son père qui a causé la guerre. Magnus n'est qu'un instrument qui permet de rétablir les normes mises à mal par son père. Ainsi, emprisonné dans la tour du château de Stockholm, il est traité selon son rang :

Je pense qu'ils le nourrirent bien :

ils ne le firent pas mourir de faim ${ }^{131}$.

L'exécution permet donc d'effacer le scandale de la mort des ducs et de montrer que leurs partisans ont su rétablir l'ordre social renversé par Birger. De la même manière, l'exécution du jeune homme prend des allures de rituel courtois :

On le conduisit à Helgeandsholmen

et on étala un tapis.

Il s'y agenouilla noblement

\footnotetext{
${ }^{128}$ I. Andersson, op. cit., p. 120-132.

${ }^{129}$ G. Vilhelmsdotter, op. cit., p. 32-33.

${ }^{130}$ Vers 4404-4405 : Som iak hauer aff herrana hört / tw giäller thz thin fader bröt. La même idée est exprimée au vers 4331.

${ }^{131}$ Vers 4332-4333 : full wäl wente jak at the han födha / ekke swelto the han til dödha.
} 
et on lui fit couper la tête.

Ils le placèrent ensuite sur une litière.

Avait été préparé un baldaquin

qu'ils placèrent sur la litière au-dessus de son corps,

car son père était un roi puissant ${ }^{132}$.

Le motif du tapis a pu être interprété en liaison avec le tabou du sang royal qui ne devait pas toucher le sol $^{133}$, mais ce motif permet aussi de montrer la différence entre les nouveaux maîtres du pays et le roi Birger qui avait laissé ses frères mourir sur le sol, tout en bas de la tour :

La tour s'élève sur un rocher.

Une mare puante et très sale

se trouvait entre eux deux ${ }^{134}$.

Une fois de plus, l'exécution de Magnus Birgersson permet d'effacer symboliquement la faute, tout comme le baldaquin, qui symbolise le rang du supplicié. Ce motif du baldaquin apparaît à d'autres reprises, au-dessus de la tête du roi Birger qui assiste à un tournoi ${ }^{135}$, mais aussi au-dessus des ducs Erik et Valdemar, eux aussi fils de roi, dont les cadavres sont portés hors de la forteresse de Nyköping sous un baldaquin :

Ceux qui étaient à Nyköping

prirent les ducs et les portèrent

hors de la forteresse sur une litière

recouverte d'un baldaquin ${ }^{136}$.

Les deux épisodes doivent être replacés dans la perspective des descriptions des enterrements de roi ou de chevaliers qui mettent avant tout en scène la reconnaissance de leur rang social. Cependant, l'Eriskrönika ne saurait être réduite à cette revendication. La loi, lorsqu'elle peut être citée, occupe une place centrale dans la chronique. Si l'auteur a recours à ces topoi, c'est qu'il n'a pas la possibilité d'exprimer son rejet du crime de Birger d'une autre manière.

\footnotetext{
${ }^{132}$ Vers 4424-4436 : pa then helgandz holma man han ledde / ok eth täpette vnder bredde / Ther knäade han sik fagherlika aa / ther loot man hans hoffwod aff slaa / ok lagdo han sidhan a ena baar / eth baldekin til redho war / ok lagdo a barena ouer hans liik / hans fader var en konung riik.

${ }^{133}$ R. Pipping, Kommentar till Erikskrönikan, op. cit., p. 712-713.

${ }^{134}$ Vers 3972-3974: Thz torn thz stod vpa en steen / en pöol ful mykit oreen / mällan thera beggia laa.

${ }^{135}$ Vers 1435.

${ }^{136}$ Vers 4266-4269 : [T]he a Nyköpunge waro / the toko hertoghana ok baro / wt aff husith a enne baar / eth baldakin ther ower war.
} 
Lorsque l'on demande aux partisans des ducs, placés devant leurs dépouilles, qui ils veulent désormais suivre, ils répondent son fils et signifient leur rejet du roi par ces mots : «Nul ne doit tuer pour hériter ${ }^{137}$ !», qui est une référence directe aux lois $^{138}$. C'est donc la loi qui disqualifie le comportement du roi, mais celle-ci ne peut exprimer l'ampleur de son crime. Dès qu'il le peut l'auteur cite, met en vers la loi (par exemple lorsqu'il résume les lois d'edsöre, équivalent suédois de la paix du roi) et souligne, lorsqu'un souverain meurt, qu'il a donné de bonnes lois ${ }^{139}$. Pour le crime de Birger, il doit palier l'absence de loi par le recours à des comparaisons implicites. Les autres personnages que l'on voit mourir de faim dans la chronique sont les Suédois partis en croisade vers l'est qui meurent du scorbut et de faim dans la forteresse de Landskrona et finissent assiégés et tués par les Russes. Or Birger est pire que ces Russes, que les Suédois appellent des païens. Il insiste sur les sévices infligés aux ducs :

Leur souffrance était terrible et cruelle.

Si un homme les avait vus,

il aurait été touché, fût-il païen. (...)

Le roi se réjouissait de voir

leur souffrance et leurs tourments ${ }^{140}$.

Erik devient un martyr, une figure christique ${ }^{141}$, face à Birger, décrit comme un tortionnaire païen. Il ne manque pas de souligner le «miracle» dont bénéficie Erik, qui, malgré les tortures, réussit à tenir neuf jours. Cette interprétation de la mort d'Erik en terme de miracle rappelle un autre épisode de la chronique: le meurtre du roi danois Erik Plovpenning par son frère, le duc Abel. La situation est inversée, puisque le duc est le tortionnaire, mais le même schéma est à l'œuvre: comme Birger qui cherchait à reprendre les provinces confiées à son frère Erik, Abel tue son frère Erik pour s'emparer du Danemark. Le corps du roi noyé est récupéré par des pécheurs et se met à faire des miracles, sous la forme de luminaires qui le suivent partout où il est conduit. Mais le roi Abel meurt peu de temps après :

Maintenant, en enfer, il n'a plus aucun repos.

Malheur à celui qui acquiert ainsi les biens du royaume terrestre

car il endurera la souffrance en enfer ${ }^{142}$.

\footnotetext{
${ }^{137}$ Vers 4281 : thz ma engin dräpa til arff.

${ }^{138}$ R. Pipping, Kommentar till Erikskrönikan, op. cit., p. 701.

${ }^{139}$ Vers 456-473, 525-529 et 1246.

${ }^{140}$ Vers 3949-3951 et 3969-3970 : thera pina war ill ok owarkwnsam / wm nokor menniskia haffdit seet / thz ware wäl enom hedningia leet (...) thz lyste konungenom wäl ath see / Thera pina ok thera nödh.

${ }^{141}$ Le mot suédois pina utilisé pour décrire les souffrances d'Erik désigne fréquemment la Passion du Christ. Voir S. B. Jansson, Medeltidens rimkrönikor. Studier i funktion, stoff, form, Uppsala, 1971, p. 173-175.

${ }^{142}$ Vers 587-589: Nw haffuer han $i$ heluite engen lisa / we er them swa jorderikis ödh / at han skal thola i heluite nödh.
} 
L'enfer est aussi promis à plusieurs reprises au roi Birger et à ses conseillers. Tous ces éléments convergent donc pour faire de Birger un roi qui a abusé de son pouvoir et auquel il est impossible d'obéir. Mais au-delà, en soulignant l'horreur de ces tueries familiales, la chronique constitue une véritable démonstration contre le principe héréditaire et le fait qu'un bien aussi important qu'un royaume puisse changer de main sans contrôle. L'élection n'empêche pas les violences, comme le montre l'arrivée au pouvoir du roi Magnus Ladulås, qui détrône son frère Valdemar. Toutefois, l'élection donne à cet acte une sanction légale, comme le montre le fait que Magnus soit le seul bon roi de la chronique (Magnus traite, selon la chronique, son frère détrôné de manière "courtoise» en défaisant loyalement ses troupes). Il forme un contrepoint à la figure inquiétante de Birger, qui a hérité de son pouvoir et qui en abuse. Lors de l'élection de Magnus Eriksson, un des meneurs de la cérémonie, qui n'est pas identifié peut ainsi rappeler :

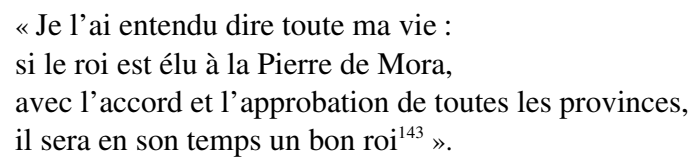

L'orateur anonyme, qui prend en charge l'opinion de la communauté, souligne non seulement la tradition de l'élection à Mora, lieu d'Uppland où, selon l'Erikskrönika, le roi Magnus Ladulås fut élu ${ }^{144}$, mais aussi sa nécessaire conformité à la loi, représentée ici par l'accord de représentants des différentes provinces. La Loi d'Uppland de 1296 est la première à décrire l'élection du roi en faisant référence à l'accord nécessaire des lagmän des provinces suédoises, mais elle ne décrit pas l'élection dans les mêmes termes de la chronique, qui est beaucoup plus précise sur la manière dont les différentes provinces doivent envoyer des délégations de paysans propriétaires (bönder). Ce n'est qu'en 1335 qu'une «ordonnance sur l'élection», dont les mesures furent reprises dans la Loi nationale rédigée au milieu du $\mathrm{XIV}^{\mathrm{e}}$ siècle $^{145}$, vint codifier de manière précise l'élection du roi, selon des modalités qui ressemblent à celles décrites dans la chronique, sans leur correspondre exactement. Bien qu'il soit difficile de dater précisément l'Erikskrönika, tout se passe donc comme si la chronique avait précédé et exprimé l'attente d'une telle loi avant 1335 ou même, en 1335, à l'occasion du tour du royaume (eriksgata) du roi Magnus Eriksson qui fut aussi l'occasion pour les habitants du Trögd de présenter leur loi.

\footnotetext{
${ }^{143}$ Vers 4556-4559: Jak hauer thz hört sakt allan myn aller / warder konunger a morasten walder / med alla landa vilia ok lagha / han skal varda goder $i$ sina dagha.

${ }^{144}$ Vers 952-957. Sur l'élection à Mora, voir E. Sjöholm, Sveriges Medeltidslagar. Europeisk rättstradition i politisk omvandling, Lund, 1988, p. 296-298. Il est possible que cette élection du roi Magnus Ladulås à Mora soit une invention de la chronique.

${ }^{145}$ Sur les lois suédoises, je renvoie à l'étude en français de Thomas Lindkvist, «Droit et genèse de l'État dans la Suède médiévale : royauté et communautés », Justice et législation, dir. A. Padoa-Schioppa, Paris, 2000, p. 251-270.
} 
L'ordonnance sur l'élection de 1335 contient une nouveauté importante: le roi est appelé à prêter serment selon des termes très précis ${ }^{146}$ et c'est en prononçant ce serment qu'il entre en pleine possession de ses pouvoirs. Dans les anciennes lois, le roi était appelé à faire le tour de son royaume (eriksgata) pour prêter serment devant les différentes assemblées (thing) de respecter leurs lois. La nouvelle ordonnance donne le texte du serment qui engage le roi devant l'ensemble des Suédois. Parmi les limites ainsi imposées au pouvoir royal, la troisième entre en résonance avec plusieurs épisodes de l'Erikskrönika :

Le troisième article est qu'il doit être pour son peuple un roi fidèle et loyal de telle sorte qu'il ne doit emprisonner ou faire emprisonner sur son ordre ou encore, d'une façon ou d'une autre, porter atteinte à personne, riche ou pauvre, avant que sa culpabilité n'ait été légalement établie, comme le dit la loi de la province où le préjudice a été commis. Il ne doit pas non plus, d'une façon ou d'une autre, leur prendre leurs biens, si ce n'est selon la loi et après un jugement légal ${ }^{147}$.

Cet Habeas corpus suédois semble répondre à plusieurs problèmes exposés par l'Erikskrönika: la figure du roi-traître, le statut et le traitement des prisonniers, l'usage de leurs biens. La Loi nationale, dans laquelle l'ordonnance fut insérée sans grands changements, se montre encore plus précise en montrant que le roi ne doit porter atteinte à personne, «dans sa vie ou dans ses membres ${ }^{148}$ ». Il faut en effet rappeler l'attention tatillonne portée par l'auteur aux conditions de détentions des personnages, la manière dont il souligne, par exemple, que Valdemar était laissé «sans fer et sans chaîne» et son fils, «libre et détaché». Il se montre particulièrement soucieux de donner des détails, sans nul doute tout aussi imaginaires, sur les ducs enfermés à Nyköping :

Bien qu'ils fussent au pilori,

ils avaient chacun une chaîne

qui était lourde et insupportable,

pesant sept lispund.

Leurs mains étaient clouées sur le devant du pilori. (...)

Leurs carcans étaient larges et épais

\footnotetext{
${ }^{146}$ Sur le serment du roi suédois, voir C. Péneau, «Separare regem a regimine regni : "Coups d'État» et expression de la loi dans la Suède des XIV et XV siècles », Coups d'États à la fin du Moyen Âge? Aux fondements du pouvoir politique en Europe Occidentale, F. Foronda, J.$\mathrm{Ph}$. Genet et J.M. Nieto Soria (éd.), Madrid, Collection de la Casa de Velázquez nº 91, 2005, p. 51-71.

${ }^{147}$ K.H. Karlsson (éd.), Södermannalagen efter cod. Havn. Ny Kgl. Saml.4 : o. N :o 2237, Stockholm, 1904, p. 27-28: Thriði articulus at han scal trygger ok troin konunger uara allum almogha sinum. sua at han angin fatøkan fanga eller richan. eller epti buði sino fanga lata. eller ok nocrom lundum fordcerua. utan han se laghlicha fyr foruunnin sum lagh sighia $i$ laghsaghu there garningin giors. ai ok thera gooz them nokraleðis af taka. utan eptir laghum ok lagha domum.

${ }^{148}$ C.J. Schlyter (éd.), Corpus iuris sueo-gotorum antiqui Samling af Sveriges gamla lagar, X-Konung Magnus Erikssons Landslag, Lund, 1862, p. 9 : a liif aella limi.
} 
et si solidement fichés au mur

qu'ils semblaient là pour l'éternité ${ }^{149}$.

Ces descriptions n'ont pas seulement pour but d'émouvoir l'auditoire, mais d'exprimer une opinion. Là où la loi manque, l'auteur doit construire une démonstration par l'action, en insistant sur les dangers d'une telle manifestation de force et sur le caractère inouï du comportement royal, que l'auditeur peut comprendre grâce aux autres épisodes mettant en scène des prisonniers. Il cherche donc à créer un consensus autour de la nécessaire limitation du pouvoir royal. Cette hypothèse d'une chronique rédigée dans le but de changer la loi n'est pas neuve. Sverre Bagge a montré que le Miroir royal (Konungsskuggsjá) avait été l'occasion pour son auteur de formuler comme des règles éthiques des principes qui n'ont été introduits qu'une dizaine d'années plus tard dans la loi norvégienne ${ }^{150}$. Howard Bloch a montré, dans son étude sur les liens entre la littérature française et les transformations des lois aux $\mathrm{XII}^{\mathrm{e}}$ et $\mathrm{XIII}^{\mathrm{e}}$ siècles, que la littérature avait participé au processus de transformation des lois et des institutions judiciaires en exposant les insuffisances et les contradictions qui naissent lorsque les lois se révèlent inadaptées aux transformations sociales ${ }^{151}$. Les débats autour de la rédaction d'une loi élective n'ont pas laissé de traces dans les sources. L'Erikskrönika est le seul document qui montre pourtant que ce débat a eu lieu et que les commanditaires de l'œuvre y ont activement participé en souhaitant faire représenter les dangers de l'hérédité et les avantages du système électif. L'introduction de la loi élective semble ainsi répondre à cette attente lorsqu'elle précise: «En Suède, le roi obtient le royaume par l'élection et non par l'héritage ${ }^{152} »$.

Cette loi élective, valable dans tout le royaume, brise le cadre provincial des lois et travaille, parallèlement à l'essor d'une législation royale depuis la seconde moitié du XIII siècle, à donner un cadre législatif commun à toute la Suède. La rédaction d'une loi commune ne s'est faite qu'au milieu du XIV siècle, mais des textes comme l'Erikskrönika ont pu servir à exprimer ce désir commun en dressant dès le prologue le cadre de son action :

[Dieu] a conçu le monde très grand :

forêts et marches, monts et montagnes,

feuilles et herbes, mer et rivage,

et de nombreux pays pleins de joie,

\footnotetext{
${ }^{149}$ Vers 3944-3948 et 3966-3968: än som han i stokkin laa / tha haffde huar thera ena boyo vpa / hon war twngh ok ekke godh / swa at hwar thera siw liuspund woogh / Thera hender waro nägelda a stokkin fram / Thera halsiern waro thiok oc breed / ok slaghin $i$ mwrin samwleedh / Som the skullo sithia ther $\ddot{a}$ oc ä. Sept lispund correspondent environ à soixante kilogrammes

${ }^{150}$ S. Bagge, The Political Thought of the King's Mirror, Odense University Press, 1987, p. 71-74.

${ }^{151}$ R. H. Bloch, Medieval French Literature and Law, Berkeley-Los Angeles-Londres, 1977.

${ }^{152}$ C.J. Schlyter (éd.), op. cit., 1862, p. 7 : Nu ar til kunungx rikit i suerike kununger valiande ok ey aruande.
} 
dont un se nomme la Suède ${ }^{153}$.

C'est aussi une définition de la Suède qui ouvre l'ordonnance sur l'élection, puis la Loi nationale :

Un royaume qui se nomme la Suède est composé de sept diocèses et de neuf provinces dirigées par un lagman, avec leurs frontières ${ }^{154}$.

Ce discours d'unité est le fait des élites, ces grands propriétaires suédois dont les intérêts économiques transcendent les divisions provinciales. L'Erikskrönika propose une vision unitaire du royaume, à travers une histoire, où chevaliers (ceux que la chronique nomme les Upplandais) et paysans sont unis. L'image de ces derniers tranche avec la vision caricaturale du paysan dans les œuvres françaises. Il est vrai que le statut des bönder suédois est radicalement différent de la position des vilains français : ils sont propriétaires de leur terre, libres, et ils peuvent aspirer à entrer dans le groupe des privilégiés, même si leur situation, à la fin du Moyen Âge, semble plutôt se dégrader ${ }^{155}$. Tous sont des propriétaires et tous sont unis face au roi, qu'il s'agisse de le renverser ou de l'élire. Comme le souligne, à propos du roi Magnus Eriksson, les derniers vers de la chronique :

La gloire qu'il eut et qu'il a encore,

il le doit aux Upplandais et aux Suédois ${ }^{156}$.

Les Upplandais sont évidemment des Suédois, mais ce nom renvoie à leur statut d'élite, sans doute car l'Uppland est un centre du pouvoir politique et religieux et une région où les grands propriétaires sont plus nombreux qu'ailleurs. La différence déterminante entre les chevaliers et les bönder, les Upplandais et les Suédois, est l'accès à des armes efficaces. Seuls les chevaliers peuvent être victorieux, ce qui est une manière de justifier, comme ailleurs, leurs privilèges ${ }^{157}$ : «les chevaliers maintenaient la paix et l'ordre ${ }^{158} »$, rappelle la chronique. Justifier l'élection du roi et demander une loi commune sous la forme d'un roman de chevalerie qui exalte les prouesses des chevaliers tout en célébrant l'union avec les paysans (bönder) est en soi un acte politique. La forme même du roman courtois contribue à exclure de la scène politique un groupe qui, tout en continuant à être nommé, voire instrumentalisé, n'apparaît pas au nombre des destinataires directs de la chronique.

\footnotetext{
${ }^{153}$ Vers 11-15 : Verldena hauer han skipat swa widha / skogh ok marka bergh ok lidha / lööff ok gräss vatn ok sand / mykin frögd ok margh land / Ok eth ther med som swerighe heter.

${ }^{154}$ K.H. Karlsson (éd.), op. cit., p. 4-5 : Eet kunungx rike sum hatir sucerike hauer i sik siu biskop ok nio laghmanz döme mep landamarum pera.

155 T. Lindkvist, «The peasantery and Peasant Communities», Campagnes médiévales. L'Homme et son espace. Étude offertes à Robert Fossier, dir. É. Mornet, Paris, Publication de la Sorbonne, 1995, p. 387-398.

${ }^{156}$ Vers 4542-4543: Then ära han fik ok hauer än / thz wlte honom gud ok vplenzske men.

${ }^{157}$ C. Péneau, Erikskrönika, Chronique d'Erik, première chronique rimée suédoise, Paris, Publications de la Sorbonne, 2005, p. 58-76.

${ }^{158}$ Vers 2020 : herrane styrkto friidh ok sät.
} 
Ce rapide aperçu ne prétend pas épuiser le sens de l'Erikskrönika, mais simplement indiquer que ce texte, dans sa globalité, peut apporter des renseignements précieux, au-delà même des détails isolés que les historiens glanent depuis plusieurs générations, sur les traces d'un débat sur la loi élective et sur la formation de la loi commune ${ }^{159}$. Dans la première des chroniques rimées suédoises, on ne trouve pas «écrit ce qui s'est passé », mais plutôt, ce qui doit advenir, le passé ne formant qu'une liste d'épisodes montrant à la fois la supériorité des chevaliers et les dangers du pouvoir royal lorsqu'ils ne peuvent le contrôler.

Écrire l'histoire sous la forme d'un roman courtois permet d'imposer un point de vue unique, celui de ses héros naturels, les chevaliers. L'historiographie naît donc en Suède de la position ambiguë de cette élite qui se distingue du groupe des propriétaires terriens - distinction inscrite dans le genre même - tout en affirmant leur solidarité, qui lie son développement à celui du pouvoir royal, tout en justifiant sa méfiance à son égard, et qui proclame sa capacité à dire le passé - et le présent - de tout le royaume, tout en montrant que seuls les «électeurs", les chevaliers du royaume, ont un rôle à jouer. En louant ces mêmes chevaliers (herra) dans son prologue, la loi de Trögd entre en résonance avec la chronique, dont on ignore, par ailleurs, la véritable diffusion au $\mathrm{XIV}^{\mathrm{e}}$ siècle. Cependant, le fait que l'Erikskrönika ait servi, au siècle suivant, de modèle historiographique à des époques de rejet du roi danois, qui était aussi roi de Suède dans le cadre de l'Union de Kalmar, montre que le sens de la chronique rimée, comme justification d'un pouvoir aristocratique, ne s'était pas perdu.

Corinne Péneau

Université de Paris XII

\footnotetext{
159 A. Musson, «Appealing to the past: Perceptions of Law in Late-Medieval England», Expectations of the Law in the Middle Ages, sous la dir. d'A. Musson, Woodbridge, 2001, p. 165-179, en particulier la conclusion: «The mythology and ideology of a people plays an equally significant part in the formation of legal policy and of the law itself and (...), through appealing to the past, members of the late medieval society sought to justify, legitimate, inform and understand their present $»$.
} 